\title{
Effects of PDE4 Pathway Inhibition in Rat Experimental Stroke
}

\author{
Fan Yang ${ }^{1}$, Rachita K. Sumbria ${ }^{2,4}$, Dong Xue ${ }^{2}$, Chuanhui $\mathrm{Yu}^{2}$, Dan $\mathrm{He}^{2}$, Shuo Liu ${ }^{1}$ Annlia Paganini-Hill², Mark J. Fisher ${ }^{1,2,3}$ \\ ${ }^{1}$ Departments of Anatomy \& Neurobiology, ${ }^{2}$ Neurology, and ${ }^{3}$ Pathology \& Laboratory Medicine, University of California, \\ Irvine, California; ${ }^{4}$ Department of Biopharmaceutical Sciences, School of Pharmacy, Keck Graduate Institute, Claremont, \\ California
}

Received, April 15, 2014; Revised, July 16, 2014; Accepted July 17, 2014; Published, July 17, 2014.

\begin{abstract}
PURPOSE: The first genomewide association study indicated that variations in the phosphodiesterase 4D (PDE4D) gene confer risk for ischemic stroke. However, inconsistencies among the studies designed to replicate the findings indicated the need for further investigation to elucidate the role of the PDE4 pathway in stroke pathogenesis. Hence, we studied the effect of global inhibition of the PDE4 pathway in two rat experimental stroke models, using the PDE4 inhibitor rolipram. Further, the specific role of the PDE4D isoform in ischemic stroke pathogenesis was studied using PDE4D knockout rats in experimental stroke. METHODS: Rats were subjected to either the ligation or embolic stroke model and treated with rolipram $(3 \mathrm{mg} / \mathrm{kg}$; i.p.) prior to the ischemic insult. Similarly, the PDE4D knockout rats were subjected to experimental stroke using the embolic model. RESULTS: Global inhibition of the PDE4 pathway using rolipram produced infarcts that were $225 \%(\mathrm{p}<0.01)$ and $138 \%(\mathrm{p}<0.05)$ of control in the ligation and embolic models, respectively. PDE4D knockout rats subjected to embolic stroke showed no change in infarct size compared to wild-type control. CONCLUSIONS: Despite increase in infarct size after global inhibition of the PDE4 pathway with rolipram, specific inhibition of the PDE4D isoform had no effect on experimental stroke. These findings support a role for the PDE4 pathway, independent of the PDE4D isoform, in ischemic stroke pathogenesis.
\end{abstract}

This article is open to POST-PUBLICATION REVIEW. Registered readers (see "For Readers") may comment by clicking on ABSTRACT on the issue's contents page.

\section{INTRODUCTION}

Stroke is a multifactorial disorder and growing evidence suggests that genetic determinants contribute to stroke risk (1). The results of the first genome-wide association study indicated that variations in phosphodiesterase 4D (PDE4D) gene are associated with increased stroke susceptibility in an Icelandic population (2). The same study suggested that inhibition of PDE4D using small-molecule inhibitors may decrease stroke risk in individuals predisposed to PDE4D polymorphisms (2). Since then, numerous replication studies in diverse population groups have been performed to determine the role of the PDE4D gene in ischemic stroke predisposition. While studies from India, Pakistan, China, and Australia have confirmed a similar association between PDE4D and ischemic stroke risk, other studies involving different population groups have failed to confirm an association (3-8). Hence, the definite role of PDE4D in stroke pathogenesis remains unresolved.
Cyclic nucleotide phosphodiesterases (PDEs) regulate cyclic adenosine monophosphate (cAMP) and cyclic guanosine monophosphate (cGMP) signaling by controlling their degradation (9-11). Among the PDE family, PDE4 specifically controls intracellular cAMP levels by catalyzing its hydrolysis (12) and pharmacological manipulation of the PDE4 pathway has been exploited therapeutically in experimental stroke $(13,14)$. Despite the growing attention on the involvement of PDE4 and its isoform, PDE4D, in ischemic stroke risk, only one experimental stroke study has attempted to assess the role of the PDE4D isoform in stroke pathogenesis. However, no causal effect between PDE4D and stroke injury has been established (15).

The present study was thus undertaken to elucidate the role of the PDE4 pathway, particularly the PDE4D isoform, in stroke pathogenesis. We

Corresponding Author: Mark Fisher, M.D., UC Irvine Medical Center, 101 The City Drive South, Shanbrom Hall 55, Room 121, Orange, CA. Email: mfisher@uci.edu 
studied the effects of global inhibition of the PDE4 pathway using the PDE4 inhibitor rolipram in both a ligation and an embolic experimental stroke model. To approximate the effects of genetic alteration of PDE4 pathway on stroke susceptibility, we gave rolipram prior to (rather than following) the ischemic insult. Specific role of the PDE4D isoform in stroke pathogenesis was determined using PDE4D knockout (KO) rats in an embolic experimental stroke model. Recently, we have shown that PDE4/PDE4D regulates brain microvascular fibrinolysis in vitro (16). In the present study, we studied the effect of PDE4 pathway inhibition on brain microvascular fibrinolysis in vivo, using immunofluorescence and gene expression microarray.

\section{MATERIALS AND METHODS}

\section{Animals:}

Adult male Fisher-344 rats weighing 200-250 grams and adult male Wistar rats weighing 300-400 grams (Charles River Labs, Hollister, CA) and adult male PDE4D knockout Fisher-344 rats, 10-12 weeks old, were used. PDE4D knockout rats were created using DNA transposon insertional mutagenesis (Transposagen, Lexington, KY). The Institutional Animal Care and Use Committee at the University of California at Irvine approved the animal protocol in accordance with NIH guidelines.

\section{Drug preparation and administration:}

Rolipram (Fisher Scientific, Fairlawn, NJ) was dissolved in vehicle (2\% DMSO in distilled water), at a concentration of $1 \mathrm{mg} / \mathrm{ml}$. Both drug and vehicle were pre-dissolved, individually packed, and kept at $-20^{\circ} \mathrm{C}$. Individual containers were coded by a researcher not involved in other experimental procedures. Animals were equally divided into experimental and control groups. Either drug (3 $\mathrm{mg} / \mathrm{kg}$ ) or vehicle was given i.p. to rats 30 minutes before the onset of ischemia for ligation model and 60 minutes before the onset of ischemia for embolic model. The time of treatment administration differed for the two models due to difference in the time required for surgical preparation and induction of experimental stroke. Inner ear temperature was measured at the time of i.p. dosing, after brain ischemia and reperfusion, and before sacrifice.

\section{Ligation model:}

Male Fisher-344 adult rats were initially anesthetized with $3 \%$ isoflurane in a mixture of $30 \%$ oxygen and $70 \%$ air. Isoflurane level was reduced to $1.5 \%$ for maintenance of anesthesia throughout surgery. A ligation model of focal ischemia was performed as previously described (17) with modifications. Briefly, both right and left common carotid arteries (CCAs) were isolated and loose ligatures were placed around each of them. The middle cerebral artery (MCA) was ligated with a 10-O ligature at the point proximal to the rhinal fissure, and interruption of blood flow was verified visually. MCA was ligated distal to lenticulostriate arteries. Three to four hours after preparative surgery, both CCAs were clamped for 1 hour under brief anesthesia (less than 5 minutes). Similar duration of anesthesia was used at the end of ischemia to allow removal of clips on the CCAs. Rats subjected to the ligation model were used for infarct analysis as described below. Fisher-344 rats were used for the ligation model since previous studies have demonstrated consistent infarct formation using this rat strain (18).

\section{Embolic model:}

For the embolic model, male Wistar rats were initially anesthetized with $3 \%$ isoflurane in a mixture of $30 \%$ oxygen and $70 \%$ air. Isoflurane level was reduced to $1.5 \%$ for maintenance of anesthesia throughout surgery. PDE4D knockout Fisher-344 rats (Transposagen, Lexington, KY) weighing 250-350 grams were also subjected to embolic stroke, with wild-type Fisher-344 rats used as controls. Embolic stroke was induced as previously described (19). Briefly, femoral arterial blood from a donor rat was withdrawn into $20 \mathrm{~cm}$ of PE-50 tubing and incubated at $37^{\circ} \mathrm{C}$ for 2 hours and then incubated at $4^{\circ} \mathrm{C}$ for 22 hours. The modified PE-10 tubing with the pre-formed clots was advanced towards the origin of MCA through external carotid artery (ECA) and internal carotid artery (ICA). Blood clots were injected into the ICA through the carotid catheter with $5 \mu 1$ saline. Since the embolic stroke model was first established using the Wistar rats and since then has been widely performed on Wister rats, we used this specific strain for embolic stroke in the present study (20). The Fisher-344 rats were used to study the effect of the PDE4D isoform on embolic stroke since this is a readily available knockout rat strain.

Arterial blood gases, blood pressure and inner ear temperature were measured at the time of dosing, before surgery, after onset of stroke, and 
before sacrifice. Arterial blood gases were measured using Trupoint blood analysis system (ItcMed, Edison, NJ). Relative regional blood flow was measured using Laser Doppler flow meter (Perimed AB, Jarfalla, Sweden). 24 hours after onset of stroke, examinations were performed as previously described (19), and animals with score 0 and 1 were excluded. Rats subjected to embolic stroke were used for infarct analysis or mechanistic studies as described below.

\section{TTC staining:}

Animals were euthanized 24 hours after stroke onset by exsanguination under isoflurane anesthesia. The brains were then quickly removed and sectioned coronally at $2-\mathrm{mm}$ intervals and incubated in a $1 \%$ solution of $2,3,5$ - tripheny ltetrazolium chloride (TTC) at $37^{\circ} \mathrm{C}$ for vital staining. Areas not stained red with TTC were considered infarcted. Infarct areas were measured in each section using image analysis software (Image-Pro, Media Cybernetics, Inc. Silver Spring, $\mathrm{MD}$ ) and the infarct volume calculated by summing the areas in adjacent sections. Percentage of injury was calculated as percent of infarct volume to total volume of contralateral hemisphere.

\section{Immunostaining:}

24 hours after embolic stroke, rat brains were quickly frozen in isopentane pre-chilled with dry ice. $7 \mu \mathrm{m}$ thick sections were cut using cryostat and stained with primary rabbit polyclonal antibodies against either von Willebrand factor (vWF) (DAKO. Carpenturia, CA), an endothelial marker, or tissue plasminogen activator (tPA) (Molecular innovations, Novi, MI), and then incubated with FITC-conjugated sheep anti-rabbit immunoglobulin $\mathrm{G}$ (Sigma-Aldrich, St. Louis, MO) for 30 minutes at room temperature. Sections were analyzed by fluorescence microscopy.

\section{Cerebral vessel isolation:}

24 hours after embolic stroke, cortex from rats treated with rolipram $(3 \mathrm{mg} / \mathrm{kg})$ or vehicle $(2 \%$ DMSO in distilled water) was isolated. Homogenized cortex tissues were centrifuged at $1000 \mathrm{rpm}$ for $20 \mathrm{~min}$. Pellets were suspended $10 \mathrm{ml}$ $25 \%$ BSA in HBSS and centrifuged at $1000 \mathrm{~g}$ for $20 \mathrm{~min}$. Suspended vessels were put in $1 \mathrm{ml}$ $\mathrm{HBSS} / 5 \% \mathrm{FBS}$, and passed through $40 \mu \mathrm{m}$ cell strainer to isolate brain endothelial cells $(21,22)$.

\section{Western blot and PCR:}

Brain endothelial cells obtained from rats subjected to embolic stroke were used for Western blot and quantitative real-time polymerase chain reaction as previously described (23). Antibodies to occludin (BD Bioscience, San Diego, CA), claudin-5 (BD Bioscience, San Diego, CA), tPA (Molecular Innovations, Novi, MI), PAI-1 (Molecular Innovations, Novi, MI) or actin (Santa Cruz, Santa Cruz, CA) were used. The primers and probes for real-time PCR were: tPA Forward: 5' - CCCTGA CCCCGACGTACAG-3', Reverse: 5' - ATGCTT GCCGTAGCCAGAA-3' Probe: FAM - TCCCTGA CTGGACAGAGTGTGAGC-TAMRA; $\beta$-actin Forward: 5'-TTCAACACCCCAGCCATGT -3', Reverse: 5'-TGGTACGACCAGAGGCATACAG -3', Probe: FAM - CGTAGCCATCCAG GCTGTGTTG -TAMRA. RT2 Profiler PCR array system (PARN-015A, SABiosciences, Frederick, $\mathrm{MD)}$ profiling 84 genes related to endothelial cell biology and 6 housekeeping genes were used for PCR array study. Ct values of each gene were calculated and imported into PCR Array Data Analysis Portal (SABiosciences, Frederick, MD).

\section{STATISTICAL ANALYSIS}

Means between experimental and control groups were compared using Student t-tests; differences among the four temporal profile groups in the validation study were tested using analysis of variance with Tukey's method for pairwise comparisons. $p<0.05$ was considered statistically significant. Data are presented as means \pm standard error.

\section{RESULTS}

\section{Global inhibition of the PDE4 pathway using rolipram:}

In preliminary studies we investigated the temporal profile of the ligation model, with three groups of eight rats subjected to 30,60 , or 90 minutes of arterial occlusion, followed by releasing clips on both the right and left CCA. Three additional animals were included as sham operation, in which only the right MCA was permanently ligated, while the CCAs were only exposed and circled with 4-O silk suture. No infarction was found in the sham-operated animals, consistent with prior observations (24). The proportion of infarct to total volume increased with increasing minutes of 
ischemia: $1 \%, 14 \%$ and $26 \%$ in the 30,60 and 90 minute ischemia groups, respectively. The differences between the 30 and 60 minute ischemia groups and between the 30 and 90 minute ischemia groups were statistically significant, and 60 minute occlusion was chosen for the present study.

In the treatment study using the ligation model, all 18 vehicle-treated rats survived transient focal cerebral ischemia, while 3 out of 18 rolipram-treated animals died 8-20 hours after arterial reperfusion. Brain temperature, measured at inner ear, was slightly lower $\left(1.8 \pm 0.1^{\circ} \mathrm{C}\right.$ at the time of ischemia, $\mathrm{p}<0.001$ and $1.7 \pm 0.1{ }^{\circ} \mathrm{C}$ at the time of reperfusion, $\mathrm{p}<0.001)$ in rolipram-treated animals compared to vehicle-treated animals (data not shown). The mean percentage of hemisphere infarction in the vehicle-treated animals was $11 \% \pm 2$. Rats subjected to rolipram treatment prior to the onset of transient focal cerebral ischemia had a significantly higher infarct volume $(25 \% \pm 3 \%$, $225 \%$ of control, $p<0.01$, Figure 1 ).

In the treatment study using the embolic model, body temperature, blood pressure, blood gas, blood $\mathrm{pH}$ and blood flow did not differ after rolipram treatment and experimental stroke compared with pretreatment levels, while regional blood flow decreased significantly $(53 \% \pm 4 \%$ of pretreatment levels, $\mathrm{p}<0.001$ ) (data not shown). The mean percentage of cerebral infarction in 16 vehicle-treated animals was $23 \% \pm 3 \%$. The 16 rats subjected to rolipram treatment prior to onset of transient focal cerebral ischemia had significantly larger infarction $(32 \% \pm 2 \%, 138 \%$ of control, $\mathrm{p}<0.05$, Figure 2).

Immunostaining studies demonstrated similar patterns of tPA and fibrin deposition in rolipram-treated and control rats. No significant changes in TPA mRNA by real-time PCR and tPA, PAI-1, claudin-5 or occludin protein by Western blot following rolipram treatment were seen as compared with control. PCR endothelial array analysis of brain vessels of rolipram-treated rats showed no significant changes compared to control animals (data not shown).

\section{Inhibition of PDE4D isoform:}

To determine effects of the PDE4D isoforms on infarct size in experimental stroke, we used 6 PDE4D knockout rats and 6 wild-type controls. These animals demonstrated no PDE4D expression by Western blot of cerebral vascular preparation (Figure 3A). The two groups did not differ significantly in mean infarct volume measured 24 hours after onset of stroke $(20 \pm 9 \%$ vs. $18 \pm 6 \%$, Figure 3B).

\section{DISCUSSION}

Our results indicate that global inhibition of the PDE4 pathway with rolipram prior to a stroke insult increases infarct size in both the ligation and embolic rat models of experimental stroke, with increases ranging from $138-225 \%$ of control. No difference in infarct size was observed between PDE4D knockout and wild-type rats subjected to the embolic stroke model. Our observed effects of global PDE4 and specific PDE4D isoform inhibition thus suggest a role of the PDE4 pathway, independent of the PDE4D isoform, in stroke pathogenesis.

Rolipram (4 - [ 3 - (cyclopentyloxy) - 4 - meth oxyphenyl]-2-pyrrolidinone), one of the most extensively studied PDE4 inhibitors, enhances the availability of intracellular cAMP in the brain by inhibiting its hydrolysis (25). Rolipram has an $\mathrm{IC}_{50}$ of approximately $1 \mu \mathrm{M}$ and shows at least a 100-fold selectivity for PDE4 family (26). Global inhibition of the PDE4 pathway with rolipram treatment in the present study results in increased infarct size suggesting the involvement of the PDE4 pathway in stroke pathogenesis. This finding agrees with other reports that have shown that rolipram treatment exacerbated neuronal injury in experimental stroke (27-29). In addition to cerebral ischemia, reports have shown exacerbation of brain injury by rolipram treatment in other models of CNS disease $(30,31)$. One possible mechanism for exacerbation of brain injury by cAMP-stimulating agents such as rolipram is induction of compensatory upregulation of certain PDE4 isoforms (32-34) to accelerate cAMP degradation $(35,36)$. Hence, treatment with rolipram prior to ischemic insult in the present study may have caused an increase in PDE4 activity resulting in exacerbation of ischemic infarction. While there is evidence that inhibition of the PDE4 pathway by rolipram reduces expression of tPA and enhances effects of ischemia in vitro (16), we observed no difference in tPA expression or fibrin deposition in the rolipram treated group. Note that some studies have reported beneficial effects of rolipram in experimental models of stroke $(13,14)$. Contradictory effects of global inhibition of the PDE4 pathway on experimental stroke outcome 
may be due to differences in experimental models, designs and brain regions examined. Extending the survival time beyond 24 hours may be necessary to observe protective effects of global inhibition of PDE4 pathway with rolipram.

A role for PDE4D has been suggested in stroke pathogenesis and inhibition of the PDE4D isoform may decrease the risk for stroke in susceptible individuals (2). However, in the present study loss of the PDE4D isoform in knock-out rats did not produce change in infarct size compared to wild-type controls, suggesting that the PDE4D isoform is unlikely to be involved in stroke pathogenesis. This is consistent with the conflicting literature on the role of the PDE4D isoform in human stroke pathogenesis (3-8). Rolipram on the other hand is known to inhibit all forms of PDE4 including PDE4A, which is highly expressed on the brain microvasculature (15), and it is likely that isoforms other than PDE4D may be involved in exacerbation of stroke injury observed in the present study.
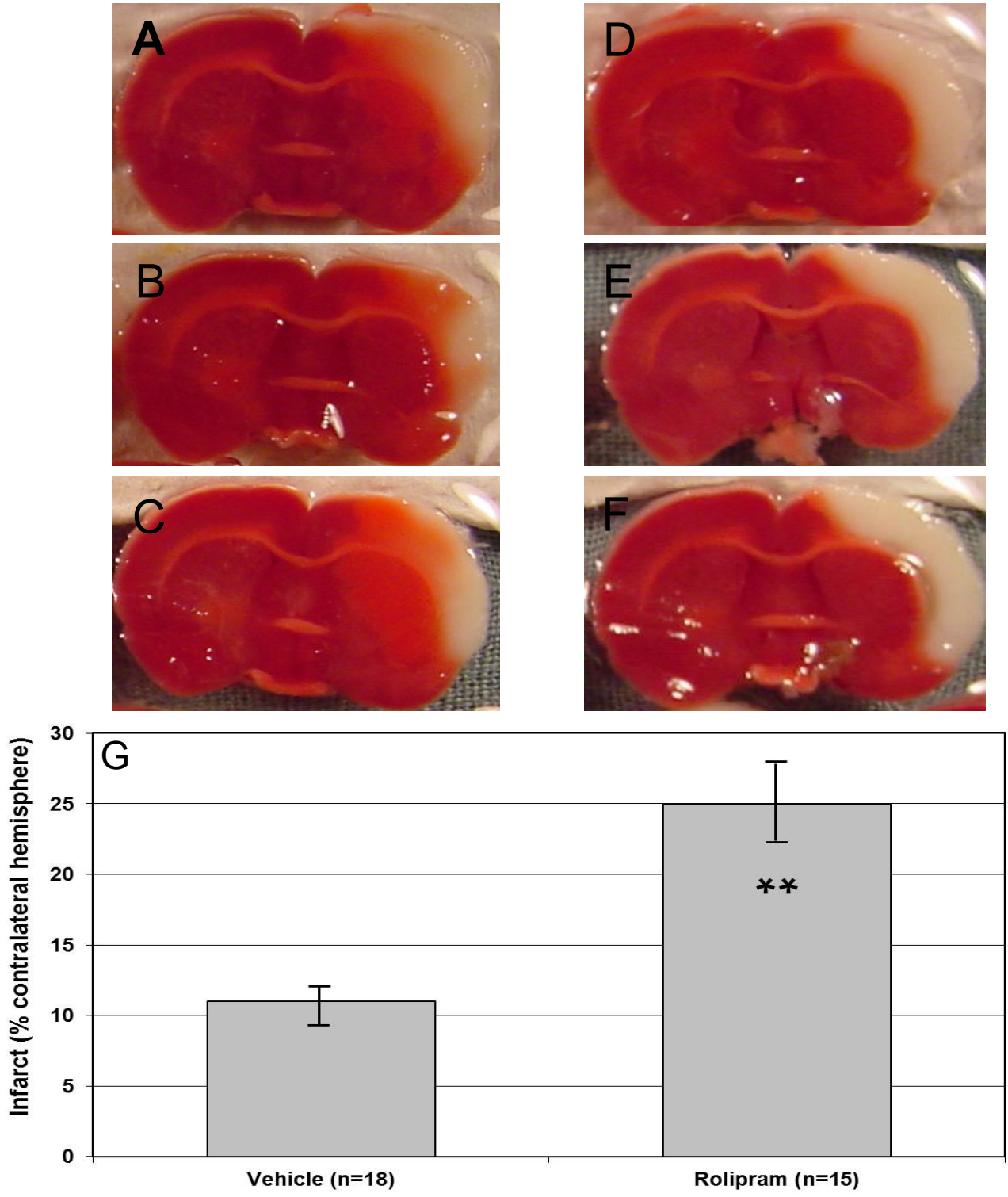

Figure 1. Coronal sections of rat brain stained with TTC 24 hours following onset of focal cerebral ischemia using ligation model. A, B, C and D, E, F are sections from vehicle $(n=18)$ and rolipram $(n=15)$ treated animal, respectively. (G) Effects of rolipram treatment on infarct size using ligation model of focal cerebral ischemia. Values are means $\pm \mathrm{SE} ; * * \mathrm{p}<0.01$. 

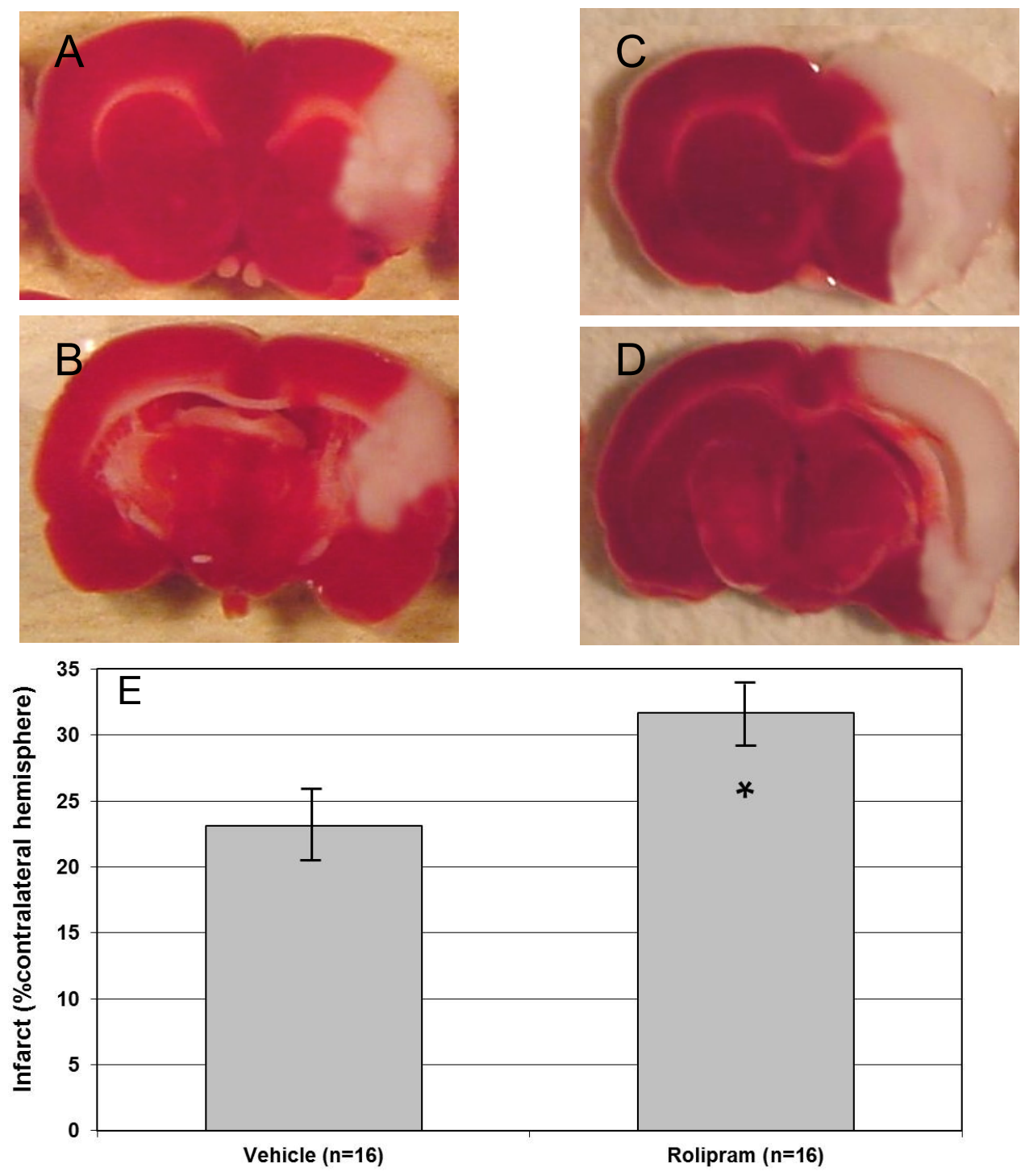

Figure 2. Coronal sections of rat brain stained with TTC 24 hours following onset of embolic stroke. A, B and C, D are sections from vehicle $(n=16)$ and rolipram $(n=16)$ treated animal, respectively. (E) Effects of rolipram treatment on infarct size with embolic experimental stroke model. Values are means \pm SE; $n=16^{*} \mathrm{p}<0.05$.

In summary, the present study shows that global inhibition of the PDE4 pathway with rolipram prior to an ischemic insult exacerbates tissue injury in two different models of experimental stroke, supporting a role of the PDE4 pathway in stroke pathogenesis. However, no evidence supporting a role of the PDE4D isoform in stroke pathogenesis was found. This suggests involvement of a different PDE4 isoform in stroke pathogenesis, and further studies are required to determine the association between modulation of other PDE4 isoforms and ischemic stroke risk.

\section{ACKNOWLEDGEMENTS}

This research was supported by the National Institutes of Health research grant NS20989. Dr. Fisher is a recipient of research support from Otsuka Pharmaceutical. 
A WT KO

PDE4D

B-actin

B

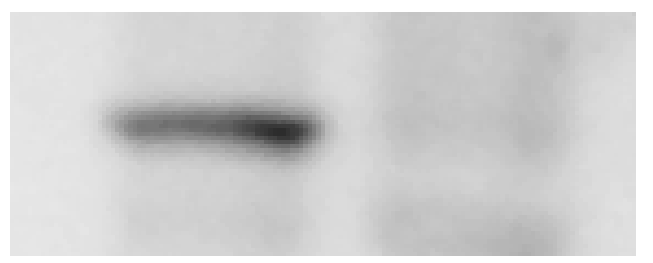

B-actin
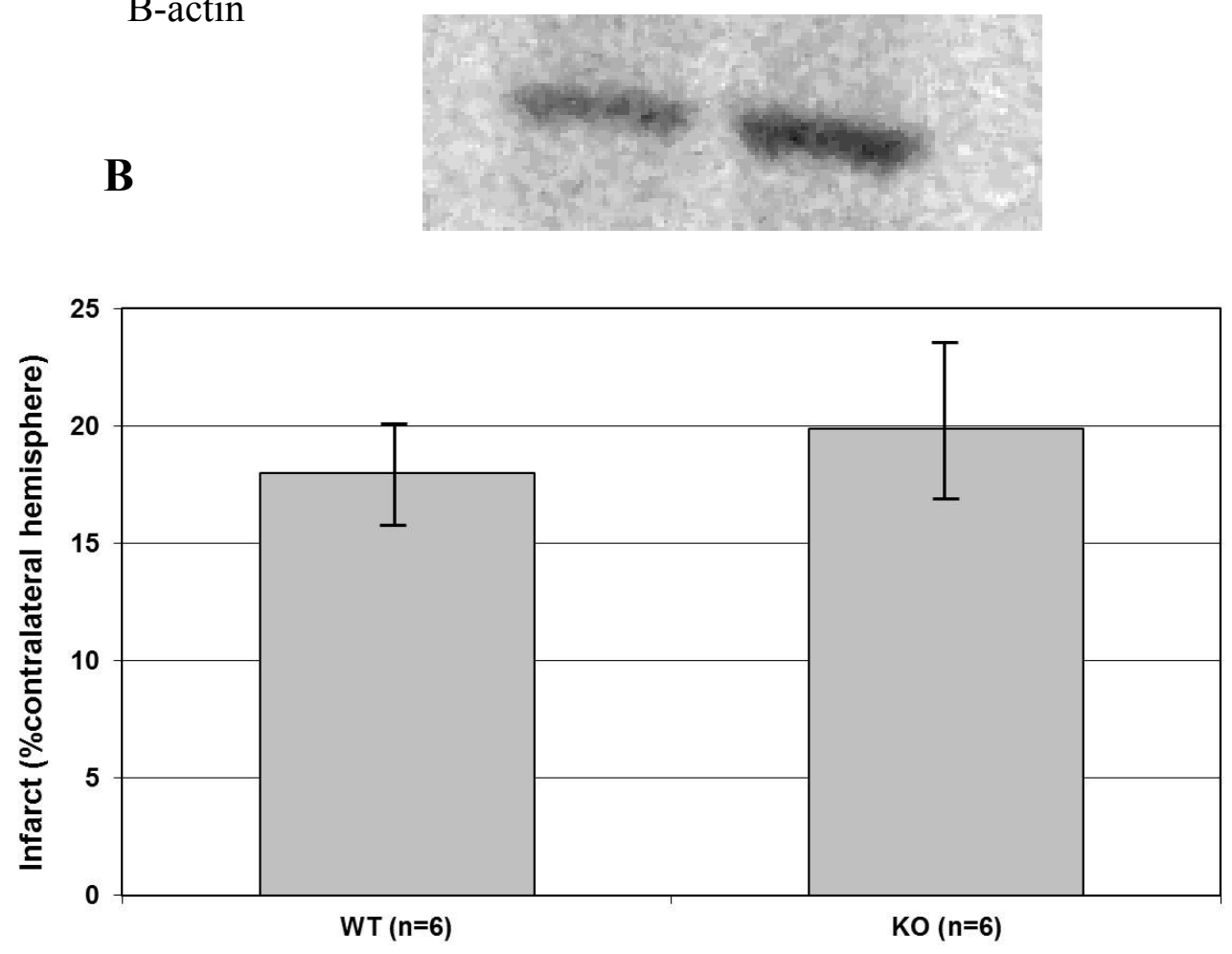

Figure 3. A. Measurement of PDE4D protein levels by Western blot of cerebral vascular preparation. PDE4D knockout rats $(\mathrm{KO})$ showed no PDE4D expression compared to wild-type rats (WT). B. Infarct size of PDE4D knockout rat $(\mathrm{n}=6)$ compared to wild-type $(\mathrm{n}=6)$ control following embolic experimental stroke model. WT: wild-type rats; KO: homozygous knockout rats. Values are means \pm SE.

\section{REFERENCES}

1. Della-Morte D, Guadagni F, Palmirotta R, Testa G, Caso V, Paciaroni M, Abete P, Rengo F, Ferroni P, Sacco RL, Rundek T. Genetics of ischemic stroke, stroke-related risk factors, stroke precursors and treatments.Pharmacogenomics, 2012;13(5):595-613.

2. Gretarsdottir S, Thorleifsson G, Reynisdottir ST, Manolescu A, Jonsdottir S, Jonsdottir T, Gudmundsdottir T, Bjarnadottir SM, Einarsson OB, Gudjonsdottir HM, Hawkins M, Gudmundsson G, Gudmundsdottir H, Andrason H, Gudmundsdottir
AS, Sigurdardottir M, Chou TT, Nahmias J, Goss S, Sveinbjörnsdottir S, Valdimarsson EM, Jakobsson F, Agnarsson U, Gudnason V, Thorgeirsson G, Fingerle J, Gurney M, Gudbjartsson D, Frigge ML, Kong A, Stefansson K, Gulcher JR. The gene encoding phosphodiesterase 4D confers risk of ischemic stroke. Nat Genet, 2003;35(2):131-138.

3. Munshi A, Babu MS, Kaul S, Shafi G, Anila AN, Alladi S, Jyothy A. Phosphodiesterase 4D (PDE4D) gene variants and the risk of ischemic stroke in a South Indian population. J Neurol Sci, 2009;285:142-145.

4. Saleheen D, Bukhari S, Haider SR, Nazir A, 
Khanum S, Shafqat S, Anis MK, Frossard P. Association of phosphodiesterase 4D gene with ischemic stroke in a Pakistani population. Stroke, 2005;36(10):2275-2277.

5. Liu X, Zhu R, Li L, Deng S, Li Q, He Z. Genetic Polymorphism in PDE4D gene and risk of ischemic stroke in Chinese population: a meta-analysis. $\mathrm{P}$ 2013 Jun 17;8(6):e66374.

6. Staton JM, Sayer MS, Hankey GJ, Attia J, Thakkinstian A, Yi Q, Cole VJ, Baker R, Eikelboom JW. Association between phosphodiesterase 4D gene and ischaemic stroke. J Neurol Neurosurg Psychiatry, 2006;77:1067-1069.

7. Matsushita $T$, Kubo M, Yonemoto K, Ninomiya $T$, Ashikawa K, Liang B, Hata J, Doi Y, Kitazono T, Ibayashi S, Iida M, Kiyohara Y, Nakamura Y. Lack of association between variations of PDE4D and ischemic stroke in the Japanese population. Stroke, 2009;40:1245-1251.

8. Kuhlenbaumer G, Berger K, Huge A, Lange E, Kessler C, John U, Funke H, Nabavi DG, Stögbauer F, Ringelstein EB, Stoll M. Evaluation of single nucleotide polymorphisms in the phosphodiesterase 4D gene (PDE4D) and their association with ischaemic stroke in a large German cohort. J Neurol Neurosurg Psychiatry, 2006;77:521-524.

9. Lugnier C. Cyclic nucleotide phosphodiesterase (PDE) superfamily: a new target for the development of specific therapeutic agents. Pharmacol Ther, 2006;109(3):366-398.

10. Conti M, Beavo J. Biochemistry and physiology of cyclic nucleotide phosphodiesterases: essential components in cyclic nucleotide signaling. Annu Rev Biochem, 2007;76:481-511.

11. Blackman BE, Horner K, Heidmann J, Wang D, Richter W, Rich TC, Conti M. PDE4D and PDE4B function in distinct subcellular compartments in mouse embryonic fibroblasts. J Biol Chem, 2011;286(14):12590-12601.

12. Alvarez R, Sette C, Yang D, Eglen RM, Wilhelm R, Shelton ER, Conti M. Activation and selective inhibition of a cyclic AMP-specific phosphodiesterase, PDE-4D3. Mol. Pharmacol, 1995;48(4):616-22.

13. Kraft P, Schwarz T, Gob E, Heydenreich N, Brede M, Meuth SG, Kleinschnitz C. The phosphodiesterase-4 inhibitor rolipram protects from ischemic stroke in mice by reducing blood-brain-barrier damage, inflammation and thrombosis. Experimental Neurology, 2013; 247:80-90.

14. Kato H, Araki T, Itoyama Y, Kogure K. Rolipram, a cyclic AMP-selective phosphodiesterase inhibitor, reduces neuronal damage following cerebral ischemia in the gerbil. Eur J Pharmacol, 1995;272(1):107-110.

15. He Z, He B, Behrle BL, Fejleh MP, Cui L, Paule
MG, Greenfield LJ. Ischemia-induced increase in microvascular phosphodiesterase $4 \mathrm{D}$ expression in rat hippocampus associated with blood brain barrier permeability: effect of age. ACS Chem Neurosci, 2012; 3(6):428-432.

16. Yang F, Liu S, Yu C, Wang SJ, Paganini-Hill A, Fisher MJ. PDE4 regulates tissue plasminogen activator expression of human brain microvascular endothelial cells. Thromb Res, 2012;129(6):750-753

17. Buchan AM, Xue D, Slivka A. A new model of temporary focal neocortical ischemia in the rat. Stroke, 1992;23(2):273-279.

18. Duverger D, MacKenzie ET. The quantification of cerebral infarction following focal ischemia in the rat: influence of strain, arterial pressure, blood glucose concentration, and age. J Cereb Blood Flow Metab, 1988;8(4):449-461.

19. Zhang RL, Chopp M, Zhang ZG, Jiang Q, Ewing JR. A rat model of focal embolic cerebral ischemia. Brain Res, 1997;766(1-2):83-92.

20. Kudo M, Aoyama A, Ichimori S, Fukunaga N. An animal model of cerebral infarction. Homologous blood clot emboli in rats. Stroke, 1982;13(4):505-508.

21. Ge S, Pachter JS. Isolation and culture of microvascular endothelial cells from murine spinal cord. J Neuroimmunol, 2006;177(1-2):209-214.

22. Nakagawa S, Deli MA, Nakao S, Honda M, Hayashi K, Nakaoke R, Kataoka Y, Niwa M. Pericytes from brain microvessels strengthen the barrier integrity in primary cultures of rat brain endothelial cells. Cell Mol Neurobiol, 2007;27(6):687-694.

23. Kim JA, Tran ND, Li Z, Yang F, Zhou W, Fisher MJ. Brain endothelial hemostasis regulation by pericytes. J Cereb Blood Flow Metab, 2006; 26(2): 209-217.

24. Coyle P. Middle cerebral artery occlusion in the young rat. Stroke, 1982;13(6):855-859.

25. Wachtel H. Characteristic behavioural alterations in rats induced by rolipram and other selective adenosine cyclic 3', 5'-monophosphate phosphodiesterase inhibitors. Psychopharmacology, 1982;77:309-316.

26. Jacobitz S, McLaughlin MM, Livi GP, Burman M, Torphy TJ. Mapping the functional domains of human recombinant phosphodiesterase 4A: structural requirements for catalytic activity and rolipram binding. Mol Pharmacol, 1996; 50(4): 891-899.

27. Tsukada H, Fukumoto D, Nishiyama S, Sato K, Kakiuchi T. Transient focal ischemia affects the cAMP second messenger system and coupled dopamine D1 and 5-HT1A receptors in the living monkey brain: a positron emission tomography study using microdialysis. J Cereb Blood Flow Metab, 2004;24(8):898-906.

28. Chang YC, Huang CC, Hung PL, Huang HM. 
Rolipram, a phosphodiesterase type IV inhibitor, exacerbates periventricular white matter lesions in rat pups. Pediatr Res, 2008;64(3):234-239.

29. Hatinen S, Sairanen M, Sirvio J, Jolkkonen J. Improved sensorimotor function by rolipram following focal cerebral ischemia in rats. Restor Neurol Neurosci, 2008;26(6):493-499.

30. Atkins CM, Kang Y, Furones C, Truettner JS, Alonso OF, Dietrich WD. Postinjury treatment with rolipram increases hemorrhage after traumatic brain injury. J Neurosci Res, 2012;90:1861-1871.

31. Atkins CM, Cepero ML, Kang Y, Liebl DJ, Dietrich WD. Effects of early rolipram treatment on histopathological outcome after controlled cortical impact injury in mice. Neurosci Lett, 2013;532:1-6.

32. Conti AC, Blendy JA. Regulation of antidepressant activity by cAMP response element binding proteins. Mol Neurobiol, 2004;30:143-155.

33. Dlaboga D, Hajjhussein H, O'Donnell JM. Regulation of phosphodiesterase-4 (PDE4) expression in mouse brain by repeated antidepressant treatment: comparison with rolipram. Brain Res, 2006;1096(1):104-112.

34. Giorgi M, Modica A, Pompili A, Pacitti C, Gasbarri A. The induction of cyclic nucleotide phosphodiesterase 4 gene (PDE4D) impairs memory in a water maze task. Behav Brain Res, 2004;154(1):99-106.

35. Campos-Toimil M, Keravis T, Orallo F, Takeda K, Lugnier C. Short-term or long-term treatments with a phosphodiesterase-4 (PDE4) inhibitor result in opposing agonist-induced $\mathrm{Ca}(2+)$ responses in endothelial cells. Br J Pharmacol, 2008; 154(1): 82 92.

36. Tilley DG, Maurice DH. Vascular smooth muscle cell phosphodiesterase (PDE) 3 and PDE4 activities and levels are regulated by cyclic AMP in vivo. Mol Pharmacol, 2002;62(3):497-506. 